\title{
Liquid Crystal-Reconfigurable Antenna Concepts for Space Applications at Microwave and Millimeter Waves
}

\author{
A. Gaebler, ${ }^{1}$ A. Moessinger, ${ }^{1}$ F. Goelden, ${ }^{1}$ A. Manabe, ${ }^{2}$ M. Goebel, ${ }^{2}$ R. Follmann, ${ }^{3}$ D. Koether, ${ }^{3}$ \\ C. Modes, ${ }^{4}$ A. Kipka, ${ }^{4}$ M. Deckelmann, ${ }^{4}$ T. Rabe, ${ }^{5}$ B. Schulz, ${ }^{5}$ P. Kuchenbecker, ${ }^{5}$ A. Lapanik, ${ }^{6}$ \\ S. Mueller, ${ }^{1}$ W. Haase, ${ }^{6}$ and R. Jakoby ${ }^{1}$ \\ ${ }^{1}$ Institute of Microwave Engineering, TU Darmstadt, 64283 Darmstadt, Germany \\ ${ }^{2}$ Merck KGaA, 64293 Darmstadt, Germany \\ ${ }^{3}$ IMST GmbH, 47475 Kamp-Lintfort, Germany \\ ${ }^{4}$ W.C. Heraeus GmbH, 63405 Hanau, Germany \\ ${ }^{5}$ Bundesanstalt für Materialforschung und -prüfung, 12205 Berlin, Germany \\ ${ }^{6}$ Institute of Condensed Matter, TU Darmstadt, 64283 Darmstadt, Germany \\ Correspondence should be addressed to A. Gaebler, gaebler@mwt.tu-darmstadt.de
}

Received 30 September 2008; Accepted 5 January 2009

Recommended by Giovanni Toso

Novel approaches of tunable devices for millimeter wave applications based on liquid crystal (LC) are presented. In the first part of the paper, a novel concept of a tunable LC phase shifter realized in Low Temperature Cofired Ceramics technology is shown while the second part of the paper deals with a tunable high-gain antenna based on an LC tunable reflectarray. The reflectarray features continuously beam scanning in between $\pm 25^{\circ}$. Also first investigations on radiation hardness of LCs are carried out, indicating that LCs might be suitable for space applications.

Copyright (c) 2009 A. Gaebler et al. This is an open access article distributed under the Creative Commons Attribution License, which permits unrestricted use, distribution, and reproduction in any medium, provided the original work is properly cited.

\section{Introduction}

The shortage in the available frequency spectrum for radio communications and the requirement for more functionality in ever smaller volume requires the development of new concepts in RF technology, especially for space missions. A general approach to solve this problem is the concept of dynamically reconfigurable RF front ends. One possibility for the realization of tunable RF components is the use of special substrate media such as controllable dielectrics. Especially for the higher frequency range (>20 GHz), liquid crystals (LCs) show very good RF performance [1]. Nevertheless, the used fabrication processes prevented a commercialization up to now, because of incompatibilities with standard fabrication processes. One aim of the Liquida project is to overcome that problem in order to develop tunable components for RF applications. The combination of both good RF properties of LC and the advantages of the standard multilayer technology of Low Temperature Cofired Ceramics (LTCC) has a high potential to enable the realization of cost-effective and highperformance RF components. Therefore, the investigation in terms of fabrication technology aims to accomplish the development of a technique for the fabrication of a Liquid Crystal compatible LTCC material system. measurement results of several LTCC phase shifter devices which can be the key component of tunable-phased array antennas. In the second part of the paper another concept of an LCtunable high-gain antenna based on a reflectarray topology is presented. Furthermore, some basic investigations for space qualification of Liquid Crystal devices are aimed for.

\section{Properties of Liquid Crystal}

LCs are an anisotropic material showing both properties of a crystal and a liquid. All further explanations are related to nematic LCs which shows up to now the best dielectric properties at microwave and $\mathrm{mm}$-wave frequencies [2]. 


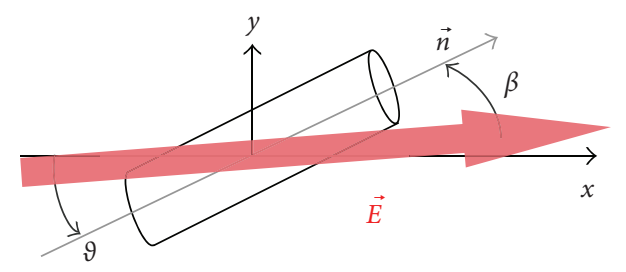

FIGURE 1: Representation of a rod like molecule with the anisotropic dielectric axes.

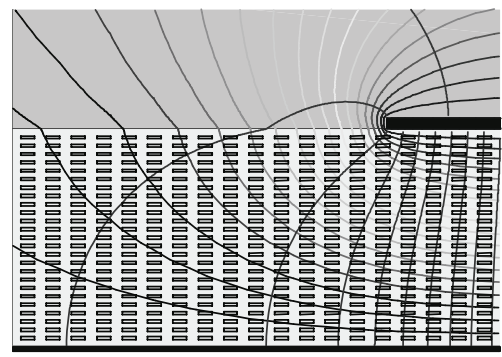

(a)

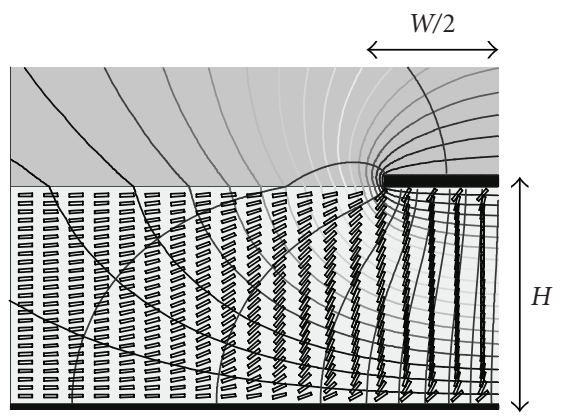

(b)

FIGURE 2: Director distribution by surface alignment without (a) and with a bias voltage on the upper electrode.

They can be described as rod-like molecules as shown in Figure 1. The shown rod represents the anisotropic dielectric properties of a molecule and the unit vector $\vec{n}$ pointing in the same direction as the rod is called director.

$\varepsilon_{r, \perp}$, the permittivity perpendicular to an incident electrical field, is usually around 2.5 while $\varepsilon_{r, \|}$ ranges between 2.8 and 3.5, depending on the material.

A bulk of LC molecules represented as such a rod contained between two surfaces which have been specially treated to anchor the molecules parallel to the surface will look like Figure 2(a).

The two electrodes in this figure represent a microstrip line and the respective ground plane. The reorientation of an entire bulk of LC can be achieved by the effect of an external electric bias field. Applying such a field, the molecule's long axis rotates such that the director points mainly towards the same direction as the exciting electric field lines. Figure 2(b) demonstrates this schematically. By using LC in such a way as a dielectric substrate, this mechanism can be used for a continuous tuning of the effective permittivity of transmission lines.

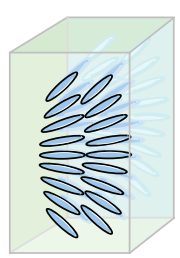

(a)

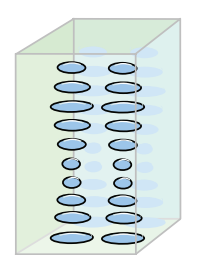

(b)

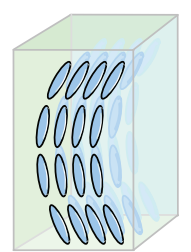

(c)
Figure 3: (a) The splay, (b) twist, and (c) bend deformation.

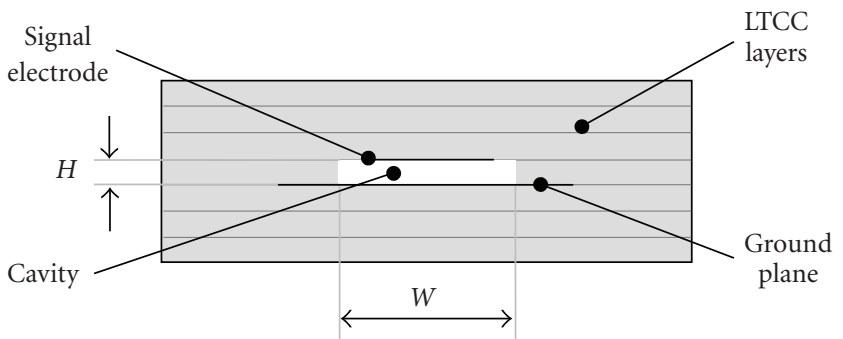

FIGURE 4: Cross-section of an LTCC-based inverted microstrip line.

To describe the director orientation in the biased state, it is necessary to consider the influence of the director dynamics which is described by the so called LC Continuum theory [3]. Looking at the rod-representation as shown in Figure 1, an electric bias-field generates an anisotropic polarization in the LC material. This leads to an electric torque for the considered director given by the cross-product of the bias field with the polarization.

Another torque contribution results from the elastic forces between the molecules themselves. These elastic forces stem from deformations of the director distribution and can conveniently be imagined to be working like deformations in a regular crystal. In liquid crystal bulks generally three basic types of deformations can exist simultaneously: the splay, twist and the bend deformation. The nature of these types is shown in Figure 3.

After the bias voltage is removed these torques lead the molecules back to the original state, caused by the minimum energy principle.

\section{Fabrication Process}

The realization of phase shifters based on Liquid Crystal combined with LTCC requires the fabrication of cavities which can be filled with LC. Figure 4 shows schematically the cross section of an inverted microstrip line (IMSL) consisting of a ground plane on the bottom of the center cavity and a top electrode. This structure is stacked here by using six layers of LTCC. The challenge is to fabricate a wide cavity or channel (e.g., dimensions of $W=400 \mu \mathrm{m}$ by $H=100 \mu \mathrm{m}$ height) and maintaining these dimensions also over a channel length of, for example, $50 \mathrm{~mm}$.

The LTCC manufacturing process uses a screen printing to generate metalized circuit patterns on so called green sheets which make the LTCC layers. Afterwards, the sheets are 


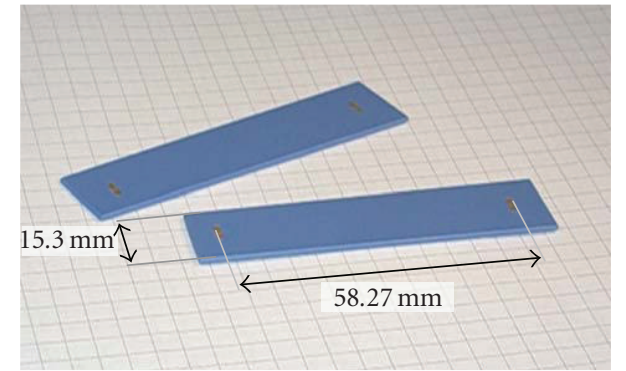

Figure 5: Prototype of a LTCC phaseshifter device.

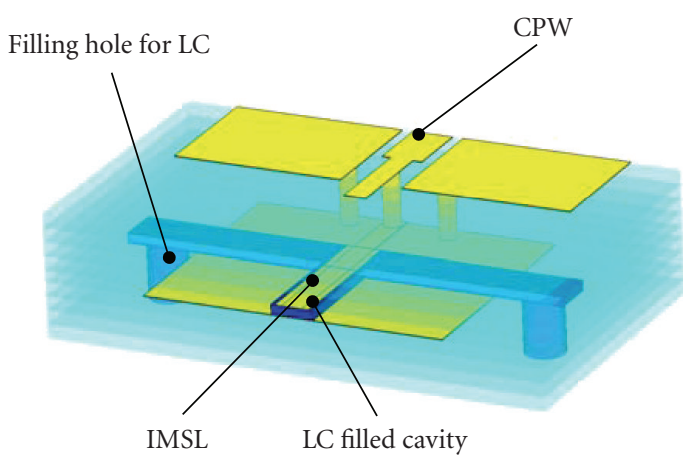

FIGURE 6: LTCC structure of the CPW to IMSL transition.

stacked and laminated by utilizing a combination of multiple lamination steps which also include forming rectangular cavities in the process.

Although the structuring of planar LTCC foils, which are processed and screen printed with thick-films, is straight forward, major problems arise such as delamination or bending of the cavity's top and bottom wall during the sintering process. Therefore, the investigation in terms of the fabrication is focused on the optimization of the process parameters needed to create integrated and well-defined cavities in LTCC.

After the burnout and co-firing process the LTCC device is filled with Liquid Crystal and finally sealed. The whole device is shown in Figure 5.

\section{LTCC Phaseshifter}

Figure 6 shows the prototype of a LTCC phase shifter schematically. A coplanar waveguide (CPW) is used for the external connection, whereas an inverted microstrip line (IMSL) realizes the liquid crystal filled transmission line phase shifter. This line type provides a high tuning efficiency [4] in the meaning of a big change of the effective permittivities in the steered and unsteered case. After applying an electrical bias field to the CPW center electrode (which has a galvanic connection to the top electrode of the IMSL), the LC can be continuously steered depending on the applied voltage as described in Section 2.

4.1. Measurement Results. The following measurement results where obtained by using the standard nematic LC

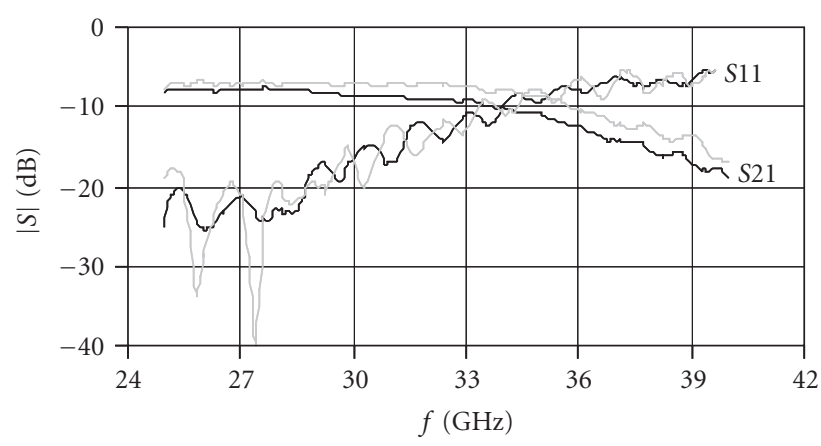

FIGURE 7: Measurement results of the LTCC phaseshifter, without bias voltage (black curves).

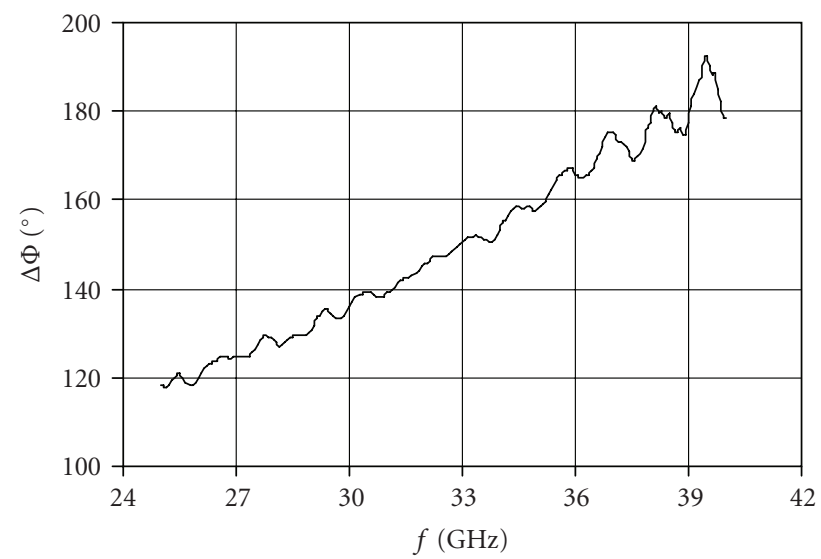

FIgURE 8: Measured phase shift.

TABLE 1: Material parameters of K15 and MDA-03-2838.

\begin{tabular}{lcccc}
\hline Mixture & $\varepsilon_{r, \|}$ & $\varepsilon_{r, \perp}$ & $\tan (\delta)_{\|}$ & $\tan (\delta)_{\perp}$ \\
\hline K15 & 2.85 & 2.55 & 0.01 & 0.03 \\
\hline
\end{tabular}

K15. This commercial available LC is well investigated and its microwave properties are also known at the targeted operation frequencies. These parameters are summarised in Table 1.

The results of the measured insertion loss $S 12$ and the attenuation are shown in Figure 7.

As depicted in Figure 7, the insertion loss of the whole devise is better than $-8.5 \mathrm{~dB}$ in the range of 25 to $30 \mathrm{Ghz}$ and the reflection is lower than $-15 \mathrm{~dB}$ for both the biased and unbiased state.

The phase shift $\Delta \Phi$ which can be achieved by using K15 is between $120^{\circ}$ and $200^{\circ}$ in the measured range from 25 to $40 \mathrm{GHz}$ (Figure 8).

In order to compare this results an quality factor of a phaseshifter as commonly called Figure-of-Merit (FoM), can be defined by the relation of the phase shift to the highest insertion loss at the corresponding frequency. Figure 9 shows the FoM which could be achieved with the presented structure. 


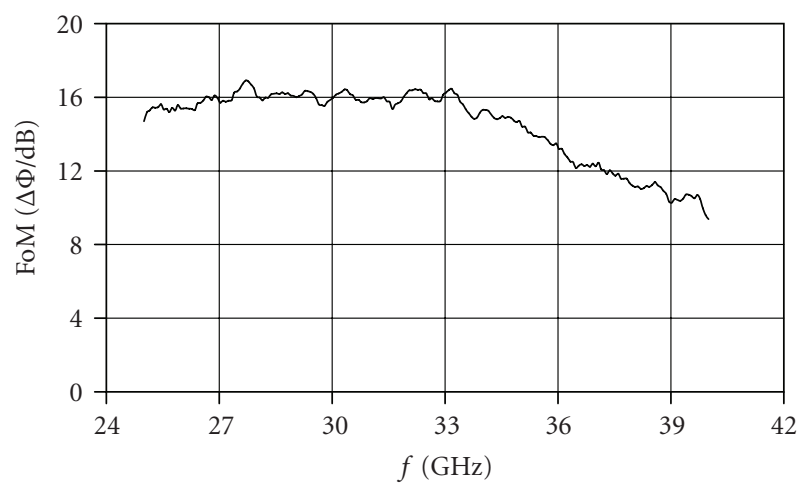

Figure 9: FoM of the LTCC phaseshifter.

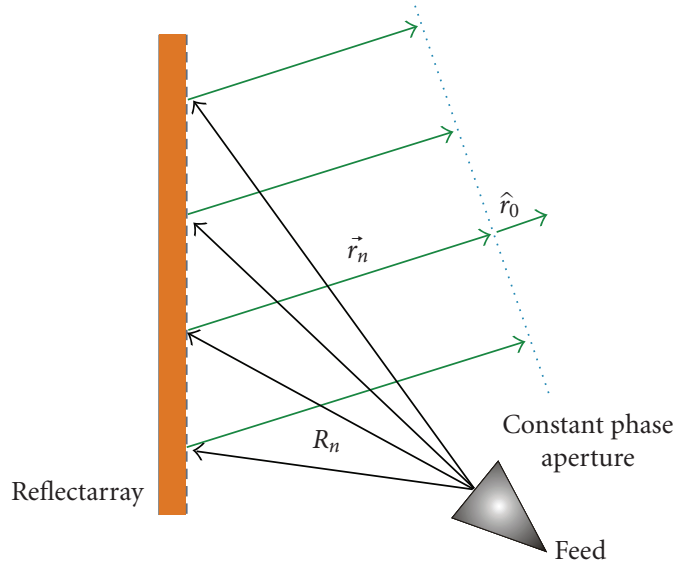

Figure 10: Functioning of a microstrip reflectarray.

\section{Space Qualification Tests of LC}

In order to verify the capability of LCs for space applications, different basic investigations have to be carried out: one test scenario is the reaction of the material under exposing of high energetic protons.

The tests has been carried out at the Forschungszentrum Jülich.

The energy of the protons is fixed to $42 \mathrm{MeV}$ with a maximum current of $10 \mu \mathrm{A}$. This energy was reduced to $15 \mathrm{MeV}$ by using a special graphite iris between the target and the proton gate in order to simulate the typical total radiation dose of a 15 years lifetime space mission in the Low Earth Orbit.

Several test cells have been irradiated with protons, for example, LTCC packaged phase shifters, LC-filled capacitances and pure LC-filled glass tubes each with four different LC mixtures.

The results of the measured values of the capacitances before and after several equivalent years of a space mission are shown in Table 1. The data was obtained by using an LCR meter at $1 \mathrm{KHz}$.

As can be seen, the reaction depends on the kind of the LC. Big changes of the conductivity could be obtained for K15 and M_A mixtures, whereas no change of those
TABle 2: Capacitance and conductivity values before and after proton irradiation.

\begin{tabular}{lcccc}
\hline \multirow{2}{*}{ Sample } & \multicolumn{2}{c}{ After irradiation } & \multicolumn{2}{c}{ before } \\
& C/pF & R/MOhm & C/pF & R/MOhm \\
\hline K15-1 & 826,5 & 6,4 & 825 & 26 \\
K15-2 & 836,0 & 6,3 & 834 & 29,2 \\
M_A-1 & 927,5 & 2,9 & 909,5 & 22,3 \\
M_A-2 & 775,9 & 3,6 & 759,5 & 30,2 \\
M_B-1 & 220,3 & 138,5 & 216 & infinite \\
M_B-2 & 229,4 & 182,0 & 225 & infinite \\
M_C-1 & 153,9 & infinite & 151,4 & infinite \\
M_C-2 & 158,0 & infinite & 157,4 & infinite \\
\hline
\end{tabular}

properties could be measured for the M_C mixture. However, the physical interpretation of all measurements is not finished yet and further tests with electrons and gamma radiation are in planning.

\section{Reflectarrays}

In place of phase shifters to realize a tunable-phased array another possibility to realize tunable high-gain antennas is a reflectarray antenna.

A reflectarray antenna combines, as its name already suggests, some of the best features of reflector and array antennas. A special type of reflectarray is the microstrip reflectarray. It consists in a basic form of a planar, thin substrate with microstrip antenna elements, for example, patches or dipoles, which are arranged in an equidistant grid on the substrate. The array is illuminated by a feed antenna and the elements of the array, in the following called "unit cells", are designed to scatter the incident wave with an appropriate phase, required to form a planar phase surface in front of the aperture, as depicted in Figure 10.

This mode of operation is similar in concept to a parabolic reflector that naturally forms a planar phase front, when a feed is placed at its focus.

One of the key features in microstrip reflectarray implementation is how the elements are designed to scatter with the desired phase. One method is to use microstrip patches with different sizes to control the backscattered phase, as proposed in [5]. This method is also the basic functionality of the tunable unit cell presented in this contribution.

Reflectarrays have attracted increasing attention in the past years because of their properties: low loss due to the absence of a corporate feed, ease of fabrication, planarity, and low weight. Reconfigurability is another desirable feature of reflectarrays that would increase their versatility a lot. A novel approach based on nematic LCs will be presented in this contribution in order to realize an electronically reconfigurable reflectarray.

6.1. Tunable Unit Cell. There have been various attempts to achieve electronic reconfigurability of reflectarrays: some of them made use of varactor diodes [6] in order to change the reflection phase, others used tunable materials, such as 


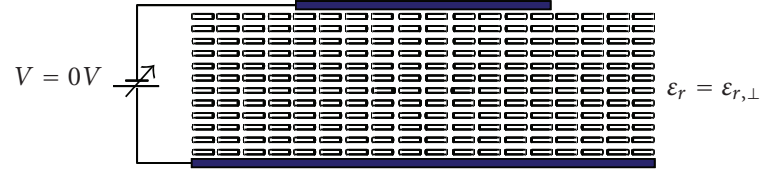

(a)

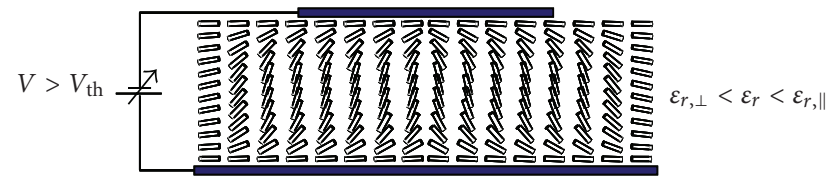

(b)

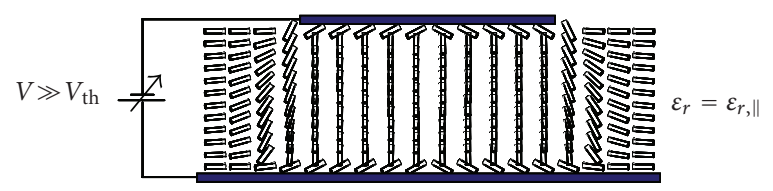

(c)

FIGURE 11: Functioning principle of the LC-tunable reflectarray unit cell with single microstrip patch.

Barium Strontium Titanate (BST) [7]. With the advances in the field of MEM-switches, these have become preferred to varactor diodes due to their superior RF properties $[8,9]$.

The novel approach, presented in this paper, makes use of a substrate which dielectric properties can be tuned by applying a low bias voltage $(<20 \mathrm{~V})[10,11]$. Thus all patches will have the same geometrical size, while the effective permittivity of the substrate under each patch will differ causing different electrical dimensions of the patches. This results in different backscattered phases which can be electronically tuned independently for each unit cell. One material that provides this functionality is LC. Because of the anisotropy of the molecules in combination with an orientational order, a macroscopically uniaxial anisotropic permittivity is effective [12] as aforementioned.

Using LCs as tunable substrate introduces several constrains in the antenna design. As the name already suggests, LCs are fluid and hence carrier substrates are required to support the metallization and the ground plane. Additionally, the carrier substrates have to form a cavity with a well-controlled thickness in which the LC can be filled in later on. The cavity has to be properly sealed to avoid LC leakage. On the ground plane and on the metallization layer a thin polyimide film has to be spin coated to provide the initial alignment of the LC director. Since the surface alignment mechanism is only functional for thin LC layers, the cavity thickness should not be much larger than $100 \mu \mathrm{m}$. This will seriously limit the bandwidth of the microstrip structure, especially for lower frequencies. Hence, the operating frequency of the reflectarray presented in this work is situated in the E-band at $77 \mathrm{GHz}$.

In Figure 11 a schematic of the cross section of a LCtunable reflectarray unit cell is presented. The cell consists of a patch printed on a carrier substrate, the ground plane printed on a carrier as well, and the LC cavity between patch and ground. A thin polyimide film (about $300 \mathrm{~nm}$ ) is spin coated both on the ground plane and on the patches, cured and finally mechanically rubbed, in order to provide the prealignment of the LC molecules.

The director of the LC-molecules is initially aligned parallel to the patch and ground, owing to the polyimide layer (Figure 11(a)). The applied bias voltage between patch and ground generates the external electric field for reorientating the director $\vec{n}$.

The RF field, given by the microstrip patch fundamental mode is mainly confined in the LC volume and is essentially perpendicular to the director. Thus, the RF field will perceive an effective permittivity $\varepsilon_{r \text {, eff }}=\varepsilon_{\perp}$. Applying an increasing bias voltage, the director will begin to rotate as soon as the voltage exceeds a certain threshold voltage $V_{\text {th }}$ of a few volts (Figure 11(b)). When the bias voltage is increased even further, the director of the LC molecules will tend to align with the bias field lines, until the molecules are completely aligned parallel to the bias Efield. In this state, the average direction of the main axes of all molecules become parallel to the RF-field and the experienced effective permittivity becomes $\varepsilon_{r \text {, eff }}=\varepsilon_{r, \|}$ (see Figure 11(c)). The change in relative effective permittivity, $\Delta \varepsilon_{r \text {, eff }}$ produces a change in the capacitance per unit length of the microstrip patch. This leads to a shift in the resonance frequency, $\omega_{r}$, and also to a shift of the phase characteristic as depicted in Figure 12. Since the permittivity variation is continuous, so is the phase tuning at a certain frequency.

6.2. Tunable LC-Reflectarray at $77 \mathrm{GHz}$. The reflectarray designed for an operating frequency of $77 \mathrm{GHz}$ has been briefly presented in [13]. It consists of 16 times 16 unit cells with an interelement spacing of $0.55 \lambda$. The size of a single unit cell is $2.2 \mathrm{~mm}$. From [14], it can be recognized that the single patch unit cell with an LC cavity height of $50 \mu \mathrm{m}$ has the best overall performance regarding losses and tunable phase range. Hence, this unit cell topology is used to realize the reflectarray.

For the substrate carrying the microstrip patches a glass carrier and for the substrate with the ground plane a metalized silicon wafer is employed. The rigidity of these materials ensures a sufficient precision for the $50 \mu \mathrm{m}$ cavity.

Spherical spacers having a diameter of $50 \mu \mathrm{m}$ are mixed together with glue, which is then used to bond the two substrates together. The glue also acts as a seal for the liquid crystal cavity.

Figure 13 shows a photograph of the fully assembled LC reflectarray with feed mounted for measurements. The feed is an open WR-10 waveguide, which is bended to radiate on the reflector.

To simplify the bias control of this first demonstrator, all patches on one row are connected altogether with a thin bias line. Therefore, the same bias voltage will be applied to the entire row and hence this reflectarray is only steerable in one plane. For this reason, there will be no beam focusing on the nonsteerable plane, the $\mathrm{H}$-plane.

The cavity has been filled with the LC mixture BMW10 developed at TU Darmstadt for maximum optical 


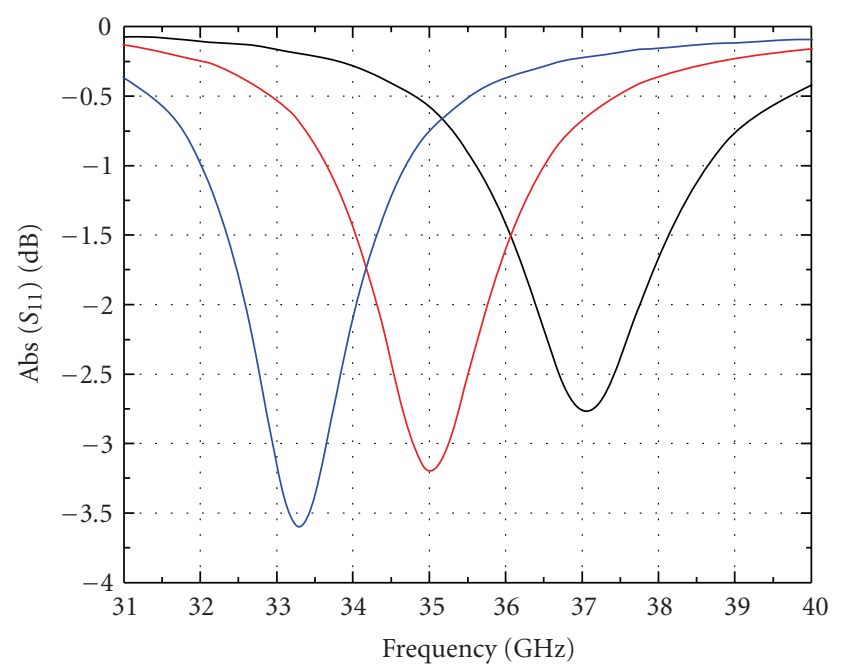

$-\varepsilon_{r 1}$
$-\varepsilon_{r 2}$
$-\varepsilon_{r 3}$

(a)

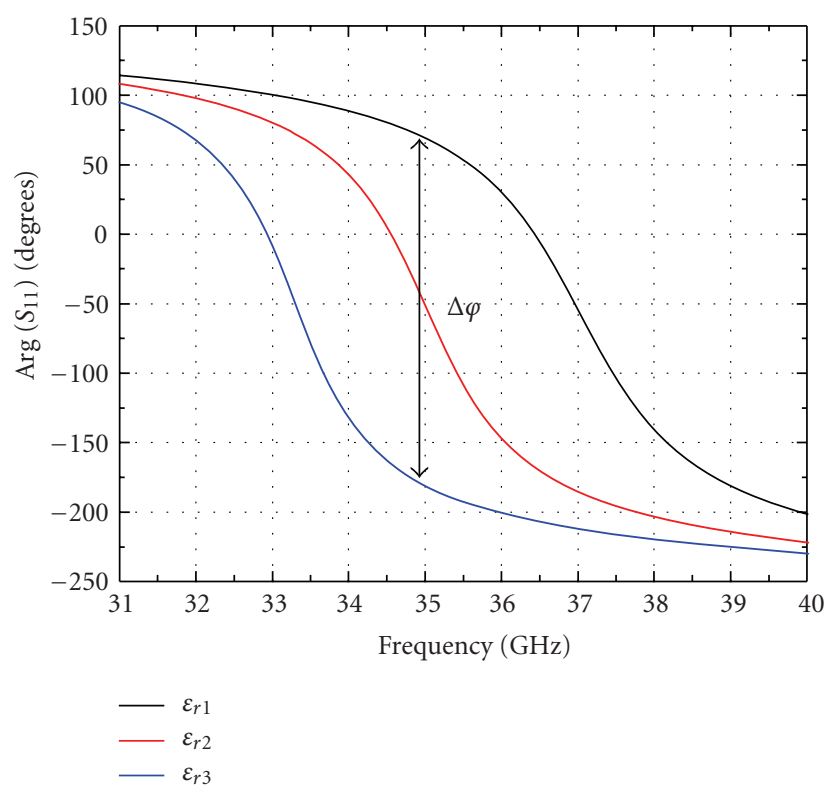

(b)

FIGURE 12: Functioning principle of a LC-tunable unit cell.

anisotropy, but featuring also acceptable microwave performance. Its characterization with the cavity perturbation technique at $30 \mathrm{GHz}$ yielded for $\varepsilon_{r, \perp}=2.49$ and $\varepsilon_{r, \|}=3.18$. The dielectric losses are $\tan (\delta)_{\perp}=0.017$ and $\tan (\delta)_{\|}=$ 0.004 .

The power pattern measurements shown in Figure 14 were performed at $77.2 \mathrm{GHz}$, this is being the frequency where the maximum adjustable phase range of the unit cells was recorded.

In order to illustrate the beam steering capability in the E-plane of the reflectarray, three selected patterns are presented: the main beam directed toward $-10^{\circ}, 0^{\circ}$, and $25^{\circ}$, respectively. The main beam of the reflectarray can

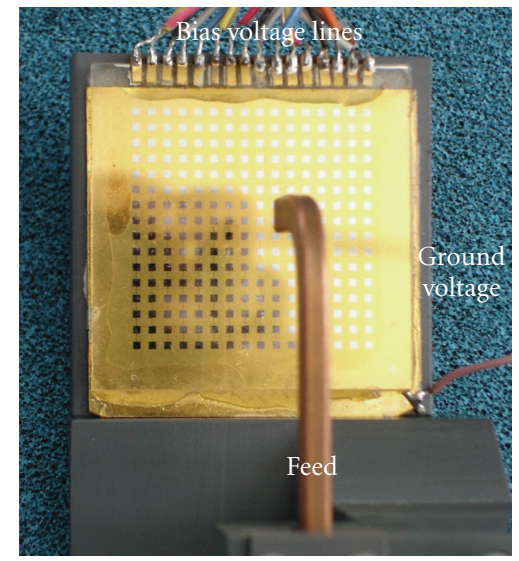

Figure 13: Fully assembled 77 GHz LC reflectarray with feed.

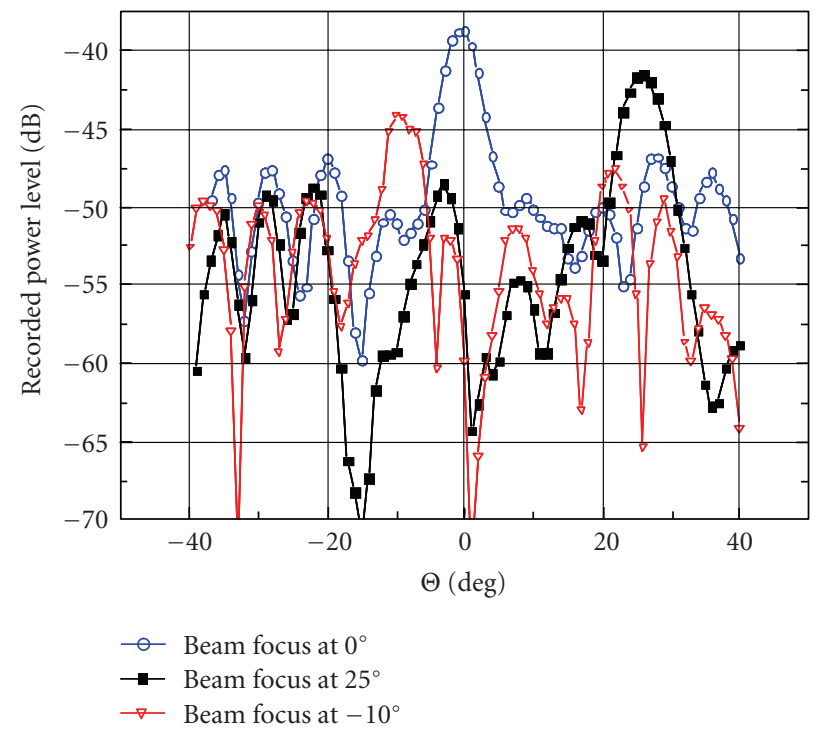

FIGURE 14: Three recorded E-plane patterns for three different voltage configurations, with the main beam pointing at different angles: $-10^{\circ}, 0^{\circ}$, and $+25^{\circ}$ (at $77.2 \mathrm{GHz}$ ).

be continuously scanned between $-25^{\circ}$ and $+25^{\circ}$ with a side lobe level of $8 \mathrm{~dB}$ for the main beam steered to $0^{\circ}$. Nevertheless, it can be observed that the pattern for the main beam directed to $-10^{\circ}$ is deteriorated in comparison to the other two. This can be caused by the beam-focusing algorithm, which evaluates only a very limited number of phase/amplitude distributions from the totality of all possible combinations [10]. It is thus not guaranteed that the obtained pattern is the best in terms of gain or side lobe level.

\section{Summary}

In this contribution, recent results of frequency agile components based on LC are presented. The first section of this contribution deals with tunable phase shifters which are the key component of tunable-phased arrays. Special attention has been paid to develop a technique for the fabrication of 
an LC-compatible LTCC material system. The phase shifters presented in this work reached a tunable phase shift of $138^{\circ}$ at $30 \mathrm{GHz}$ with a FoM of 16 . It should be noted that these first measuring results can be considerable improved by using an LC which is optimized for microwave applications as shown in [1].

In the second part of the paper, a demonstrator of a tunable reflectarray antenna has been presented. With this topology, it is possible to realize tunable high-gain antennas. The realized demonstrator has been designed to operate at $77 \mathrm{GHz}$ and is able to steer the main beam continuously in between $\pm 25^{\circ}$ by applying bias voltages below $15 \mathrm{~V}$. Its performance could be improved by employing LCs with better microwave features as employed in [14] and by improving the configuration algorithm calculating the bias voltages.

As there are at least some LC mixtures available which prove to be stable in the exposure of protons, tunable devices based on LC seem to be suited to be used in space applications, especially as for this concept no moving mechanical parts are needed.

\section{Acknowledgments}

The authors are thankful for CST GmbH for providing the CST Microwave Studio solver. The work presented in this paper has been supported in the frameworks of the Liquida, a project funded and supported by the German Aerospace Center (DLR) and the Federal Ministry of Economics and Technology (BMWi), and by the DFG Project Rekonfigurierbare Millimeterwellen-Antennen mit steuerbaren hochanisotropen Flüssigkristallen.

\section{References}

[1] S. Mueller, P. Scheele, C. Weil, M. Wittek, C. Hock, and R. Jakoby, "Tunable passive phase shifter for microwave applications using highly anisotropic liquid crystals," in Proceedings of IEEE MTT-S International Microwave Symposium Digest, vol. 2, pp. 1153-1156, Fort Worth, Tex, USA, June 2004.

[2] S. Mueller, Grundlegende Untersuchungen steuerbarer passiver Flüssigkristall-Komponenten für die Mikrowellentechnik, Ph.D. dissertation, FG Funk-Kommunikation, TU Darmstadt, Darmstadt, Germany, April 2007.

[3] J. Anderson, P. E. Watson, and P. J. Bos, LC3D: Liquid Crystal Display 3-D Director Simulator Software and Technology Guide, Artech House, Boston, Mass, USA, 2001.

[4] A. Gaebler, F. Goelden, S. Mueller, and R. Jakoby, "Efficiency considerations of tuneable liquid crystal microwave devices," in Proceedings of the German Microwave Conference (GeMIC '08), pp. 271-274, Hamburg, Germany, March 2008.

[5] D. M. Pozar, S. D. Targonski, and H. D. Syrigos, "Design of millimeter wave microstrip reflectarrays," IEEE Transactions on Antennas and Propagation, vol. 45, no. 2, pp. 287-296, 1997.

[6] S. V. Hum, M. Okoniewski, and R. J. Davies, "Realizing an electronically tunable reflectarray using varactor diode-tuned elements," IEEE Microwave and Wireless Components Letters, vol. 15, no. 6, pp. 422-424, 2005.

[7] R. R. Romanofsky, J. T. Bernhard, F. W. van Keuls, F. A. Miranda, G. Washington, and C. Canedy, "K-band phased array antennas based on $\mathrm{Ba}_{0.60} \mathrm{Sr}_{0.40} \mathrm{TiO}_{3}$ thin-film phase shifters," IEEE Transactions on Microwave Theory and Techniques, vol. 48, no. 12, pp. 2504-2510, 2000.

[8] H. Legay, G. Caille, E. Girard, et al., "MEMS controlled phase shifter elements for a linear polarized reflectarray," in Proceedings of the 28th ESA Antenna Workshop, Noordwijk, The Netherlands, May-June 2005.

[9] R. Sorrentino, R. V. Gatti, L. Marcaccioli, and B. Mencagli, "Electronic steerable MEMS antennas," in Proceedings of the 1st European Conference on Antennas and Propagation (EuCAP '06), pp. 1-8, Nice, France, November 2006.

[10] A. Moessinger, R. Marin, S. Mueller, J. Freese, and R. Jakoby, "Electronically reconfigurable reflectarrays with nematic liquid crystals," Electronics Letters, vol. 42, no. 16, pp. 899-900, 2006.

[11] W. Hu, M. Y. Ismail, R. Cahill, et al., "Liquid-crystal-based reflectarray antenna with electronically switchable monopulse patterns," Electronics Letters, vol. 43, no. 14, pp. 744-745, 2007.

[12] P. Collings and M. Hird, Introduction to Liquid Crystals: Chemistry and Physics, Taylor \& Francis, London, UK, 1997.

[13] R. Marin, A. Moessinger, F. Goelden, S. Mueller, and R. Jakoby, "77 GHz reconfigurable reflectarray with nematic liquid crystal," in Proceedings of the 2nd European Conference on Antennas and Propagation (EuCAP '07), pp. 1-5, Edinburgh, UK, November 2007.

[14] A. Moessinger, R. Marin, J. Freese, S. Mueller, A. Manabe, and R. Jakoby, "Investigations on $77 \mathrm{GHZ}$ tunable reflectarray unit cells with liquid crystal," in Proceedings of the 1st European Conference on Antennas and Propagation (EuCAP'06), pp. 1-4, Nice, France, November 2006. 

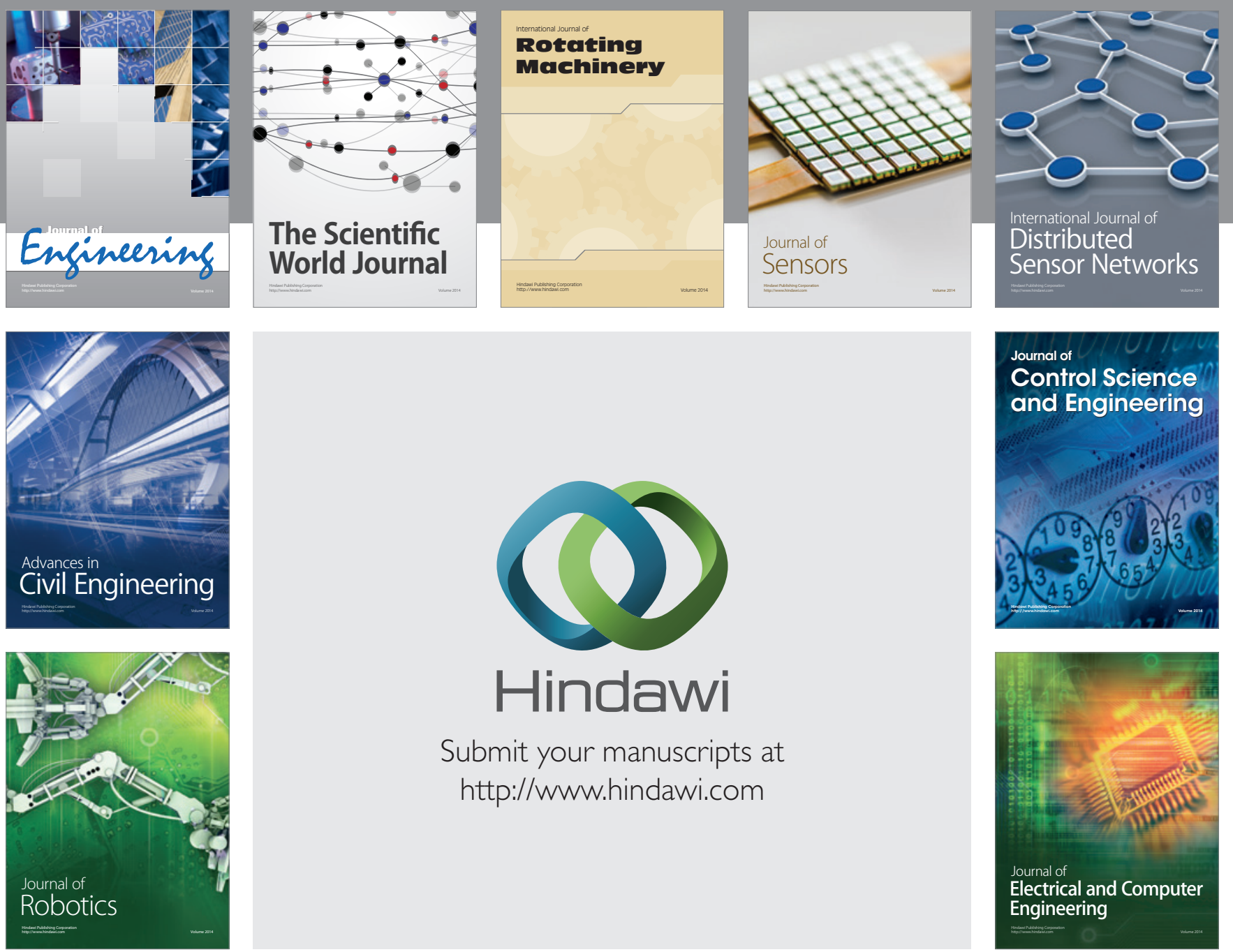

Submit your manuscripts at

http://www.hindawi.com
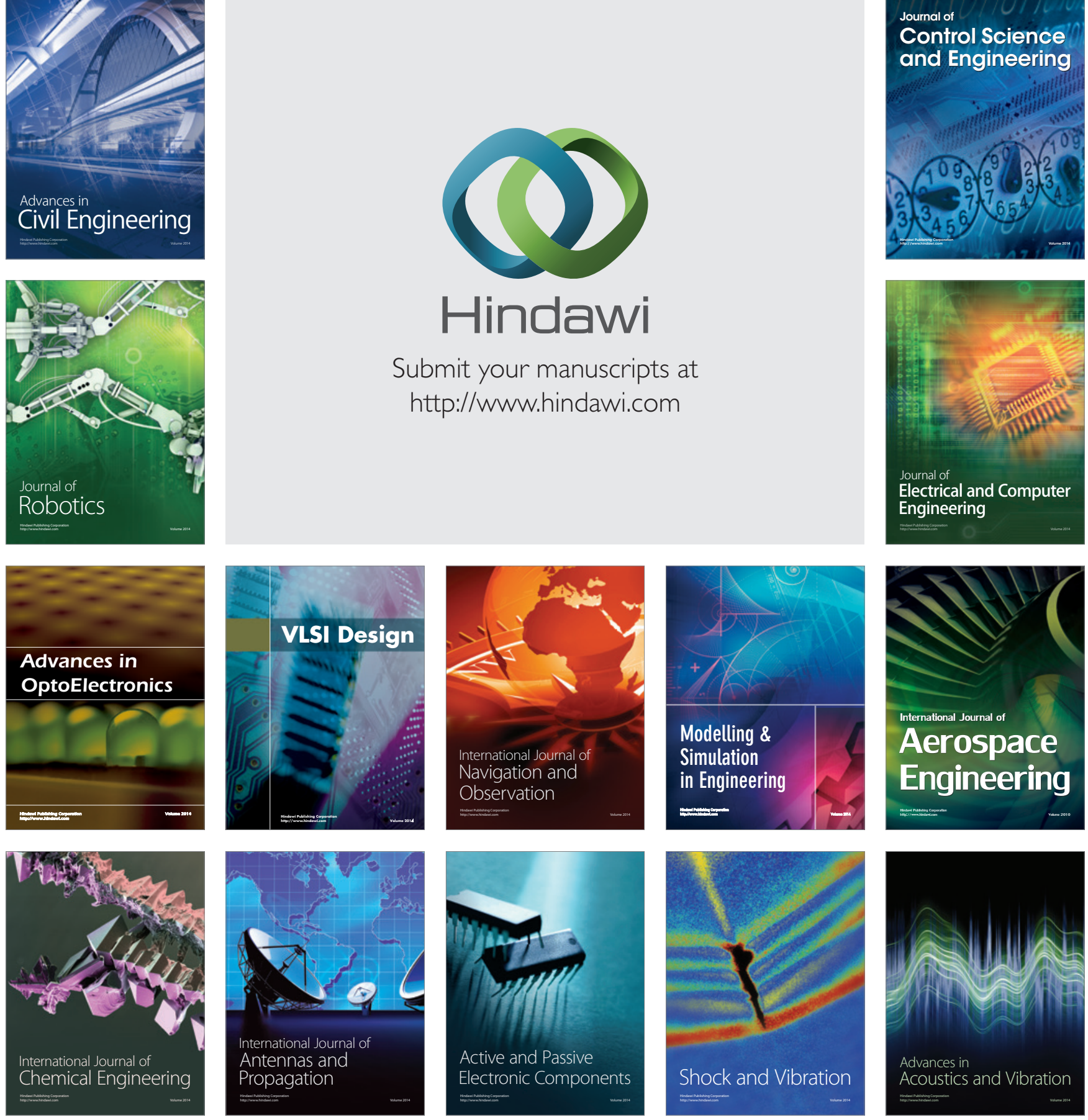